

\title{
Enhancing Broadcast Vehicular Communications Using Beamforming Technique
}

\author{
Ahmed Soua, Walid Ben-Ameur, Hossam Afifi
}

\section{To cite this version:}

Ahmed Soua, Walid Ben-Ameur, Hossam Afifi. Enhancing Broadcast Vehicular Communications Using Beamforming Technique. International Workshop on Vehicular Communications and Networking (VECON), 2012, pp.565. hal-00761075

\section{HAL Id: hal-00761075 \\ https://hal.science/hal-00761075}

Submitted on 4 Dec 2012

HAL is a multi-disciplinary open access archive for the deposit and dissemination of scientific research documents, whether they are published or not. The documents may come from teaching and research institutions in France or abroad, or from public or private research centers.
L'archive ouverte pluridisciplinaire HAL, est destinée au dépôt et à la diffusion de documents scientifiques de niveau recherche, publiés ou non, émanant des établissements d'enseignement et de recherche français ou étrangers, des laboratoires publics ou privés. 


\title{
Enhancing Broadcast Vehicular Communications Using Beamforming Technique
}

\author{
Ahmed Soua, Walid Ben-Ameur, Hossam Afifi \\ Institut Mines-Telecom, Telecom SudParis, France \\ Email: firstname.name@it-sudparis.eu
}

\begin{abstract}
A beamforming-based broadcast technique is proposed here for VANETs. It is based on two key information: the direction to the destination and the beamforming angle $\theta$. Simulations demonstrate the efficiency of our proposal in terms of probability of transmission success, ratio of implicated nodes and bandwidth gain. An analytical model is derived to calculate the forwarding transmission area. Simulation results match very well the mathematical expressions and show that the analytical model is precise.
\end{abstract}

Keywords-Broadcast, Vehicular Ad Hoc Networks (VANETs), beamforming, angle of transmission, area of transmission, bandwidth, probability of success, implicated nodes.

\section{INTRODUCTION}

Vehicular ad hoc networks are regarded as a great tool that can enhance driver safety. To efficiently implement this kind of systems, several challenges need to be tackled such as the rapidity in disseminating the alert message among the platoon of vehicles as well as the reliability guaranteed by the technique in reaching the destination. At the wireless link level, a step has been already made in the 802.11 standard [15] by accelerating the link setup (the IEEE $802.11 \mathrm{p}$ removes the need of association between stations before communicating). At the network level, one method that is considered to be very effective is broadcast. It is a technique that does not require a prior end-to-end connection establishment or maintenance. It is cheap and simple in terms of deployment and gives good performance thus its popularity.

In this paper, we are mainly interested in broadcast communications for safety applications to assist drivers in hazardous situations. We propose an Enhanced Beamformingbased Broadcast Protocol (EBBP) that combines broadcast and directional beamforming system. Relay nodes, selected by EBBP to disseminate the alert packet, are chosen based on their distance from their selector and the angle they have with it and with the destination. Our broadcast mechanism can be implemented at the physical layer with directive antennas. It is, in this case, complementary to routing in upper layers. It can be combined for example with other solutions such as the one of [12] where a geographical routing mechanism is proposed.

Another major contribution in our paper is the development of an analytical model providing a very close approximation of the total transmission area in the broadcasting scheme. Simulation results confirm the quality of the model.

\section{RELATED WORK}

A number of efforts have been already accomplished to investigate the dissemination paradigm for vehicular adhoc networks. Thus, several studies worth our special mention here.

These so-called intelligent flooding strategies can be classified into two major categories: probability based, and position based algorithms.

In probability-based broadcast techniques, each node retransmits received packets with an adjustable probability aiming at minimizing bandwidth utilization. As an exemple, in [19], three probability-based broadcast schemes are proposed: weighted p-persistence, slotted 1-persistence and slotted ppersistence schemes. They are meant to be used at the network layer and mitigate the broadcast storm problem in well connected networks. The performance of this technique was evaluated in terms of packet loss rates, overhead and reachability. Nevertheless, the major inconvenient of this approach is its inflexibility. In fact, in sparse networks, where the density of vehicles is very low, this proposal can damage the system performances since many transmissions, that would have been necessary to reach the destination, are omitted.

In order to remediate to the deficiencies of probabilitybased protocols, position-based routing protocols [3] were introduced. They assume that each vehicle is aware of the positions of other vehicles in order to select its one hop neighbor that ensures the communication between the nodes even in high mobility scenarios. This type of routing has several advantages namely the low overhead since no prior establishment and maintenance of routes is required and the good performance in highly dynamic scenarios.

Many position-based protocols have been proposed. GPSR is one example that consists of forwarding a packet to the onehop neighbor that minimizes the distance to the destination [4]. Y. Wang et al. in [5] propose an improvement of the GPSR protocol by enhancing the decision-making of data delivery. In fact, they use the concept of vector, called also Greedy mode, to choose the next relay to enhance the accuracy of the routing scheme. For intersections scenarios, they add a predictive mode to predict the motions of neighboring vehicles. Simulations reveal that their technique outperforms GPSR protocol in terms of packet delivery ratio and routing overhead. In [6], authors highlight the importance of the direction of the moving nodes on the lifetime of the links between a node and its neighbors. 
We note that the direction of the destination hasn't been taken into consideration in most of the previous algorithms. In [7] authors propose a Position-based Directional Vehicular Routing algorithm. Next hops are selected based on their angular directions relative to the destination. Note that all of the above routing algorithms try to ensure point-to-point communications and does not focus on broadcast.

Kim et al. [18] propose a neighbor knowledge-based protocol to carry out a more reliable, efficient and fast flooding technique in vehicular ad hoc networks by reducing redundant retransmissions to the minimum. Nodes apply two techniques called dynamic delay and priority checking. They have to know the distance between them and their one-hop neighbors to decentrally manage the decision whether to forward a packet or not.

In [19], three probability-based broadcast schemes are proposed: weighted p-persistence, slotted 1-persistence and slotted p-persistence schemes. They are meant to be used at the network layer and mitigate the broadcast storm problem in well connected networks. The performance of this technique was evaluated in terms of packet loss rates, overhead and reachability.

In [8], a role-based multicast protocol is proposed. It suppresses broadcast redundancy by assigning shorter waiting time prior to rebroadcasting to more distant receivers. This study focuses on achieving maximum reachability in a sparsely connected or fragmented network. To deal with the effect of node density and wireless channel quality, Slavik and al. propose a multi-hop wireless broadcast scheme for vehicular networks called DADCQ [9]. This protocol utilizes the distance method to select forwarding nodes. In addition, they adapt their decision threshold function to the density of nodes, the node clustering factor and the Rician fading parameter. DADCQ achieves high reachability and low bandwidth consumption compared to other existing multi-hop dissemination protocols.

Sun, Feng and al. in [17] proposed the TRAck Detection protocol (TRADE) which classifies the neighboring vehicles into three main categories according to their position on the road; same road-ahead, same road behind and different road. Then, the algorithm selects nodes from each group in order to forward safety messages; the farthest vehicles from same roadahead and same road-behind and all vehicles from different road are chosen to rebroadcast the alert message.

Claudio E. Palazzi and al.[10] also proposed the Fast Broadcast (FB) protocol which uses a distance-based approach with an estimated transmission range in order to reduce the number of redundant transmissions of the alert message as well as the hops to be traversed. This scheme is composed of two phases. The first one, named estimation phase, aims to provide each vehicle with an up-to-date estimation of its backward transmission range. On the other hand, the second one, called broadcast phase, is performed only when a message has to be broadcasted to all cars in the sender's area-of-interest.

In [11], authors propose a zone forwarding scheme for information dissemination in vanets $(\mathrm{ZBF})$. The proposal tackles the problem of retaining the message in the target zone for the duration of the effect time. Thus, the algorithm divides the effect area of the alert message into segments of length the transmission range. A forwarder, a vehicle which is elected in each segment, is assigned the task of broadcasting the information to other neighboring vehicles. When the forwarder is about to leave the zone, a new forwarder will be elected. Simulation results show that ZBF outperforms other information dissemination protocols.

To deal with the broadcast issue at road intersections, Tung et al. propose a directional broadcast protocol, by using precise GPS navigation system, called Efficient Road-based Directional broadcast (ERD) [13]. It groups vehicles based on their road segments (single road case, node near an intersection case and node in an intersection) and selects relay nodes for each group. The protocol improves packet efficiency significantly but it still depends on a very precise position navigation system and an accurate road digital map which are not always present on each vehicle.

Finally, in [14], the authors present an area-based broadcast technique called Location-Based Directional Broadcast protocol (LDB). It relays the alert message taking into account the position and velocity of the concerned vehicles. The authors give some heuristic rules to choose the best relay nodes.

Despite the acceptable performances, we believe that these algorithms are too complex for the context and the purpose of vehicular safety applications. Moreover, they rely on precise navigation positioning system which is not available on all vehicles.

None of the previous proposals try to derive analytical models for their system. In our study, we design a novel solution to reduce the bandwidth usage by limiting the numbers of broadcasters. Our proposal, compared to previous state of the art, is much simpler and does not require precise positioning system. Rather than using simulation, we derive analytical models for our technique to estimate performance metrics such as probability of success, bandwidth gain and implicated nodes. We believe these new models will be helpful to analyze similar problems and that on the long run, they will replace simulation tools.

\section{PROPOSED TECHNIQUE}

We suppose that a message is sent from a source to a destination in a vehicular context. The nodes (vehicles) are assumed to be randomly positioned in an area. The nodes that receive an intermediate message will broadcast it on their turn unless they have seen it before, or unless they are the destination. There is no knowledge on whether the message has reached its destination or not. This means that a node may continue to broadcast while the message has already arrived. The system ends broadcasting when there are no more relays to broadcast. In this case, we assume that the message has arrived or that the system has encountered a transmission failure. The transmission failure is a situation when there is not any intermediate relay to reach the destination.

The main feature of our protocol design is that the broadcast operation will be adjusted based on some basic geographic information and that it will use a directive beamforming system. 
The simple geographic information is composed of two parameters.

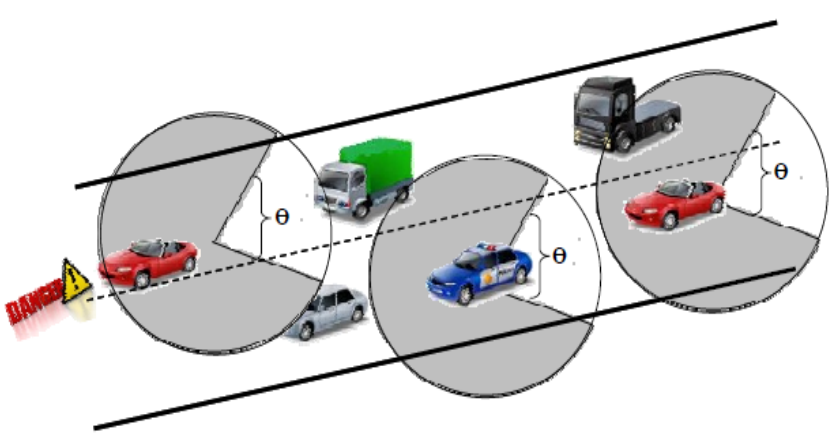

Fig. 1: Directional beamforming broadcast scheme

The first is the destination direction (it can be an approximate value). In fact, the source is supposed to know or to guess the direction of its destination. Then, our algorithm can be adapted to relatively precise or less precise direction information as it will be seen. The success or failure of the transmission is strongly dependent on the density of neighbor nodes $\lambda$.

We believe that the general information of direction in the vehicular context is very meaningful. In general, we can see that communication is either directed to the roadside or to cars coming or going in the same or in the opposite directions.

The second parameter is the beamforming angle $\theta$ (see Figure 1). This angle has a great effect on the overall performance. A very wide angle provides some insurance that the message would reach its destination. Notice that when the information related to the direction of the destination is uncertain, using a large angle provides some robustness. However, a larger set of nodes is involved and the performance of the overall relaying system might be bad.

A small beamforming angle prevents waste of bandwidth but may lead to more transmission failures.

\section{RESULTS}

In this section, we evaluate the performance of our proposed technique through MATLAB simulations. We study precisely the impact of the density of nodes and the beamforming angle on the performances of our proposal. The results confirm the efficiency of our algorithm in terms of use of bandwidth, the ratio of implicated nodes and the probability of transmission success.

In fact, transmitting messages within a limited area clearly reduces the number of nodes having to relay the message and considerably reduces the total number of transmissions and affect the probability of success. Moreover, varying the angle of transmission $\theta$ and the density of the nodes will give us an indication on the spectrum efficiency.

There will be two kinds of results in this section. Some simulation results related to the probability of success and the proportion of vehicles participating to the forwarding process. An analytical model will also be given for the second performance criteria (the proportion of implicated vehicles).

\section{A. Some simulation results}

In order to analyze the performance of our protocol, we conduct several simulations varying the following system parameters:

- Number of vehicles in the simulation from 1000 to 3000 .

○ Beamforming angle $\theta$ (from $22.5^{\circ}$ to $135^{\circ}$ ).

The nodes are assumed to be randomly positioned in a square area according to a Poisson process of density $\lambda$ (so $\lambda$ is equal to the number of nodes divided by the area of the square).

This assumption can safely be adopted since during rush hour traffic in an urban scenario the velocity of vehicles is limited and the vehicle density is very important.

Indeed, the speed of vehicles in an urban area, compared to the duration of wireless transmission, is very small and therefore we can assume that the node positions and connectivity do not change significantly from a broadcast operation to broadcast operation.

Furthermore, in such urban conditions (large density of vehicles, small inter-vehicle spacing) terrain is not a significant factor.

In Figure 2, we show a snapshot of the nodes implicated in the transmission of a message from source $S$ to destination $D$. We consider that the angle is $\theta=60^{\circ}$ and that all nodes have a transmission range $200 \mathrm{~m}$. The figure shows that the relays are located within a geographical area. The analytical model will prove that this shape is similar to a leaf.

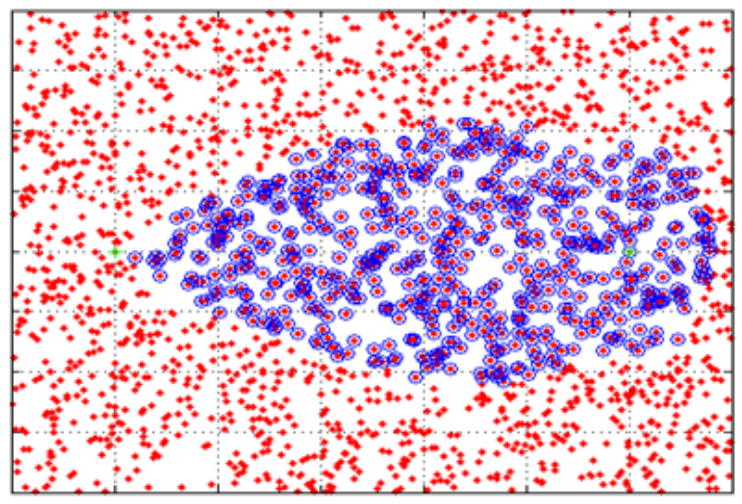

Fig. 2: Network topology and distribution of implicated nodes in the transmissions

Figure 3 gives the ratio of implicated nodes in the transmission when varying the angle $\theta$ and the total number of vehicles $n$. One can see that the two curves are close. However, the curve relative to $n$ equal to 3000 achieves slightly higher values. This observation confirms the scalability of our proposal. Clearly, the fact that we transmit messages within a limited area reduces the number of nodes having to relay the message and in turn considerably reduces the total number of transmissions.

In addition, the use of indicators to determine whether a node had already transmitted a message gives us the possibility to avoid unnecessary retransmissions and hence avoid the broadcast storms. 


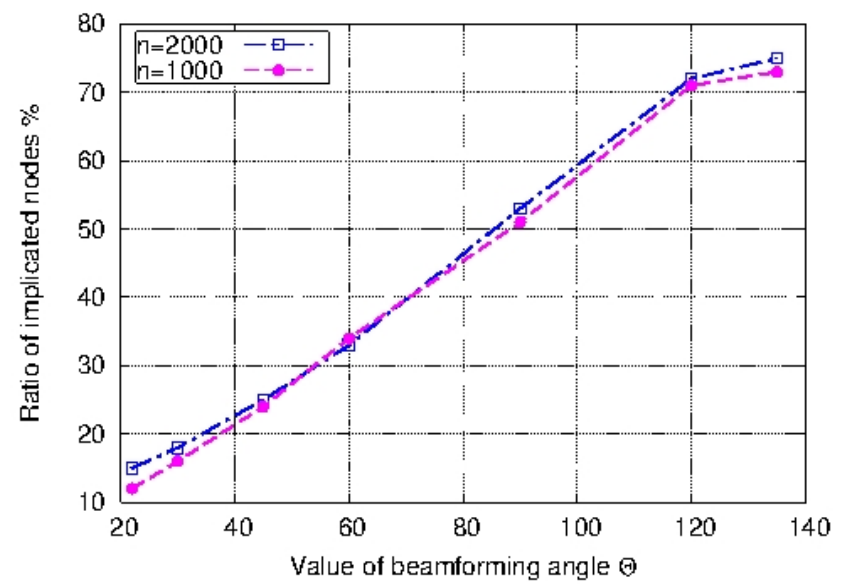

Fig. 3: Ratio of implicated nodes

Figure 4 shows the gain in bandwidth (and hence the spectrum efficiency) when varying the angle of transmission $\theta$ with a total number of nodes equal to 2000. The spectrum use ratio of relays is presented by the product of the implicated nodes' ratio and $\frac{\theta}{360^{\circ}}$.

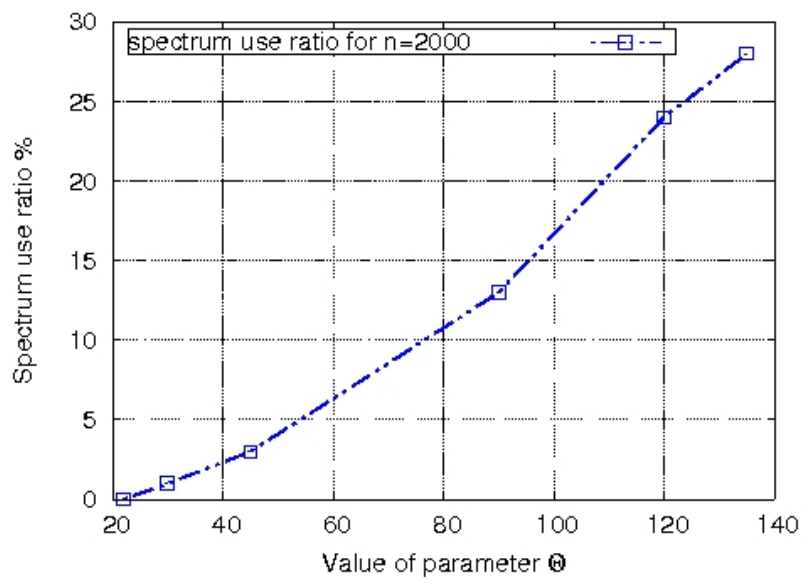

Fig. 4: Gain in bandwidth

Observe that the beamforming mechanism achieves a large gain in the spectrum use when compared to a typical broadcast operation involving all nodes.

Figures 5, 6 and 7 show the variations of the success probability with the beamforming angle $\theta$.

In each figure, different densities are considered (different node numbers). The distance $d$ between the source and the destination is equal to $1000 \mathrm{~m}$ in Figure 5 , while it equals 2000m (resp.3000m) in Figure 6 (resp.7).

Observe that the probability of success seems to be convex for small angles and concave for large angles.

One can deduce from Figures 5, 6 and 7 that the angle should be chosen carefully depending on the distance and the density.

\section{B. An analytical model for the transmission area}

In this section, we present an analytical model to estimate the transmission area (.i.e., the set of nodes that receive a

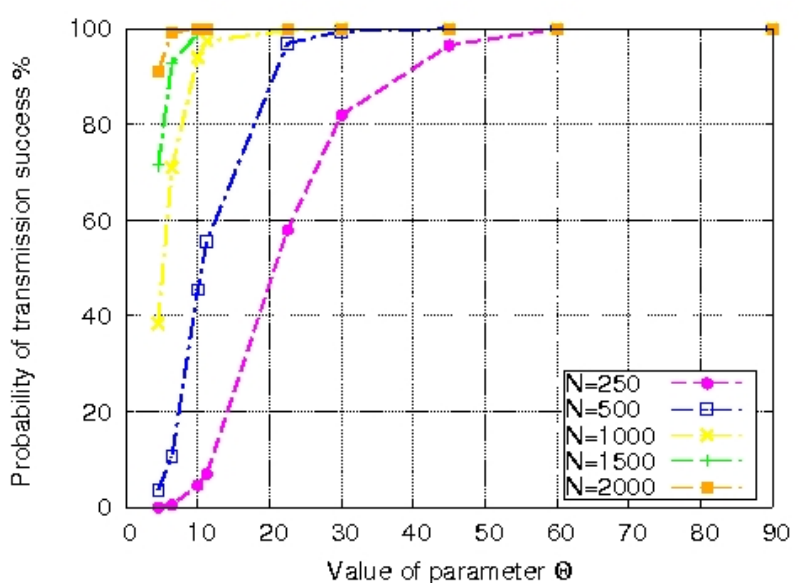

Fig. 5: Probability of transmission success $(\mathrm{d}=1000 \mathrm{~m})$

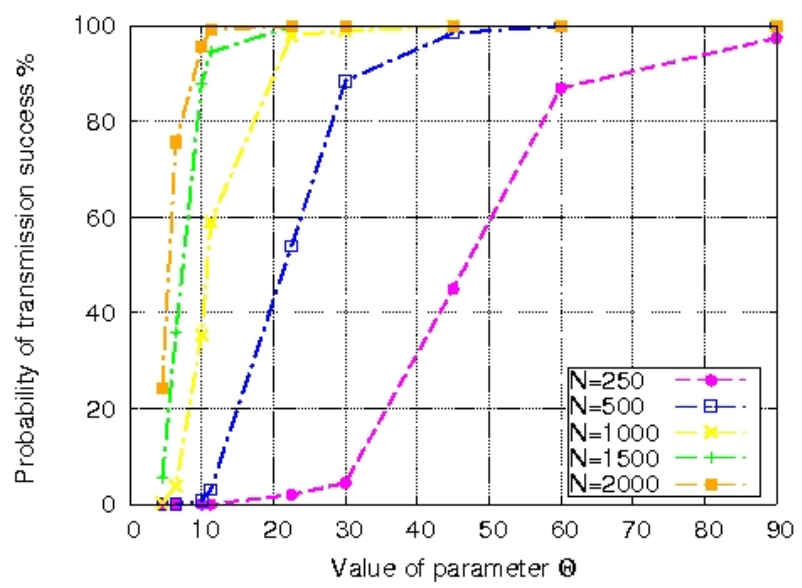

Fig. 6: Probability of transmission success $(\mathrm{d}=2000 \mathrm{~m})$

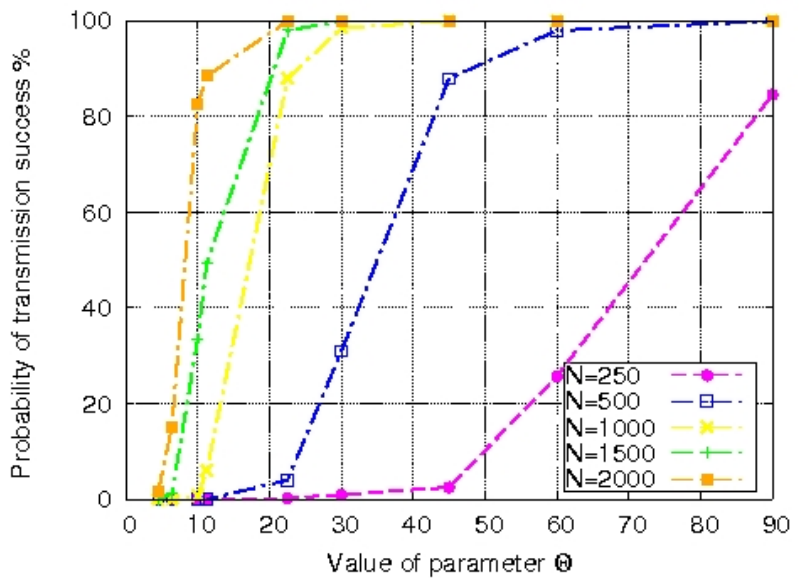

Fig. 7: Probability of transmission success $(\mathrm{d}=3000 \mathrm{~m})$ 
message and retransmit it).

We assume that nodes are uniformly distributed in a square. The source and all relay nodes transmit messages according to an angle $\theta$ within a radius $r$.

As shown on Figure 8, the transmission can be approximated by a set of triangles (the destination is a vertex of each triangle). Starting from the triangle containing the source, each two consecutive triangles have a common vertex (in addition to the destination). Each triangle's vertex is obtained by considering that there is a mobile situated in a vertex of the previous triangle transmitting within a distance $r$ according to an angle $\theta$. It should be understood that the union of these triangles is only an approximation of the true transmission area. We will see that this approximation is very good. Observe that the transmission area is again similar to a leaf.

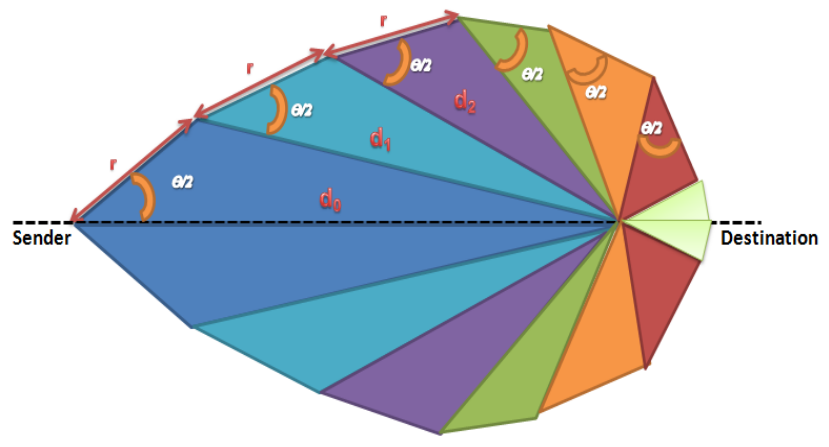

Fig. 8: Area of transmission

Since the transmission area is symmetric, we can focus on the half plane above the line connecting the source and the destination.

Starting from the triangle containing the source and using $d_{i}$ to denote the length of the common edge between the triangle number $i$ and the triangle number $i+1$, the area of triangle number $i$ is given by:

$$
S_{i}=\frac{1}{2} r d_{i} \sin \frac{\theta}{2}
$$

Moreover, the distances $d_{i}$ and $d_{i-1}$ are linked through the following equation:

$$
d_{i}^{2}=d_{i-1}^{2}+r^{2}-2 r d_{i-1} \cos \frac{\theta}{2}
$$

All these distances and triangle areas can be iteratively computed using the two previous equations. This directly leads to the total area given by:

$$
S=2 \sum_{i=1}^{N_{b}} S_{i}
$$

We evaluate hereafter the model in (3) and compare it to our simulations. The result is expressed in terms of the ratio of implicated nodes with respect to the angle $\theta$.

We show on Figure 9 the ratio of implicated nodes given by simulation when there are 1000 nodes in the square and also when there are 3000 nodes. We also show the theoretical ratio given by Eq.(3). Notice that the theoretical value does not depend on the mobiles density.
One can see that the three curves are very close. This clearly implies that the analytic model provides a very good approximation of the real situation.

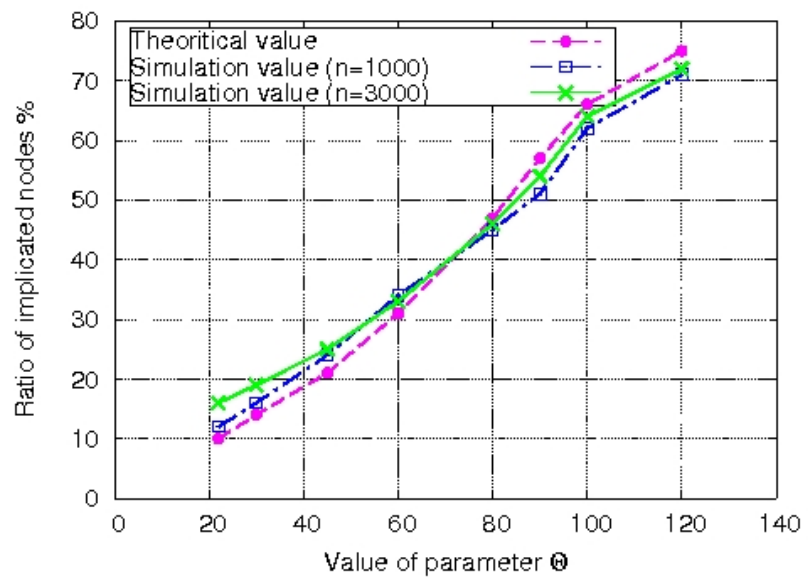

Fig. 9: Comparison between the theoretical and practical values of the implicated nodes ratio for different densities

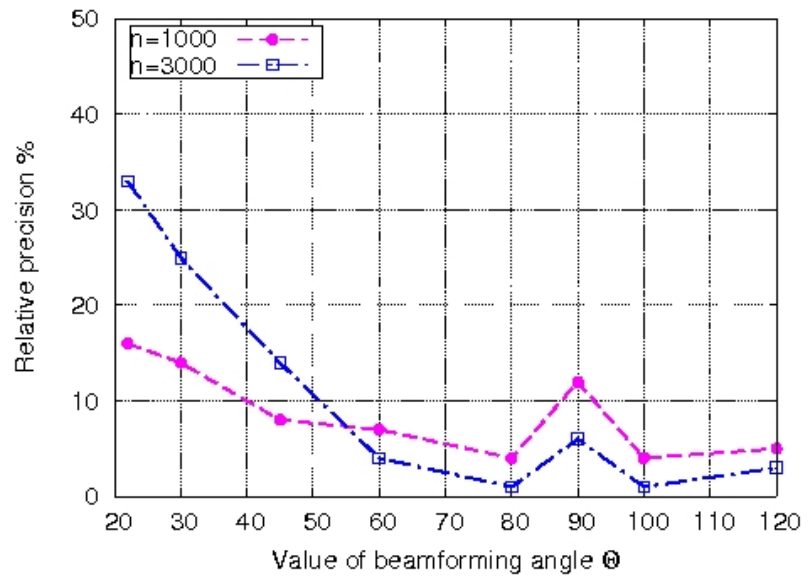

Fig. 10: Relative simulation results

Figure 10 represents the relative error of the model computed as follows:

$$
\text { relative }_{\text {error }}=\frac{\text { theoratical }_{\text {value }}-\text { simulation }_{\text {value }}}{\text { simulation }_{\text {value }}}
$$

The relative error seems to be particularly small for angles between $45^{\circ}$ and $120^{\circ}$.

An important observation related to Figure 9 (and confirmed by many other simulations) is that the ratio of implicated nodes seems to vary linearly when $\theta$ is less than $100^{\circ}$.

\section{CONCLUSION}

In this paper, we proposed a technique combining geographic routing and broadcast. The nodes located at a distance less then $r$ from the emitting vehicle and belonging to the angular sector of opening angle $\theta$ in the direction of the destination are chosen as relays. 
As mentioned before, EBBP can serve as a substrate to overlay systems. In fact, it can be applied as a prior phase of a routing technique such as ant and swarm approaches.

An analytical model was given to estimate the ratio of vehicles that will relay the message. Comparisons with simulations have clearly shown the very good precision of the model. More simulations have been conducted to estimate the probability of a message to reach the destination. This probability is high for typical values of vehicle density and distances between the source and the destination.

This combination of beamforming technique and geographic routing suggests that EBBP should be able to send alert messages with some guarantees in terms of success probability and without overloading the network and wasting bandwidth in a vehicular urban environment.

\section{REFERENCES}

[1] D. B. Johnson and D. A. Maltz, "Dynamic source routing in ad hoc wireless networks," Mobile Computing, Volume 353, 1996, pp. 153-181.

[2] C. E. Perkins and E. M. Royer, "Ad-hoc on-demand distance vector routing," in Proceedings of the 2nd IEEE Workshop on Mobile Computing Systems and Applications, 1999, pp. 90-100.

[3] E. Spaho, L. Barolli, G. Mino, F. Whafa, and V. kolici, "Vanet simulatots: A survey on mobility and routing protocols", In Proc. of the International Conference on Broadband and Wireless Computing, Communications and Applications, 2011.

[4] B. Karp and H. T. Kung, "GPSR: Greedy perimeter stateless routing for wireless networks," in Proc. of the 6th Annual ACM/IEEE International Conference on Mobile Computing and Networking, 2000, pp.243-254.

[5] Y. Wang, T. Wu, W.lee, and C.Ke, "A novel geographic routing srategy over VANET," In 24th International Conference on advanced Information Networking and Applications Workshops (WAINA), April 2010.

[6] F. Granelli, G. Boato, and D.Klizovich, "Mora: a movement-based routing algorithm for vehicle ad hoc networks", in Proc. IEEE Workshop on Automotive Networking and Applications, December 2006.

[7] D. Tian, K. Shafiee and V. C.M. Leung, "Position-based Directional Vehicular Routing," in Proc. IEEE GLOBECOM, Crown, 2009.

[8] L. Briesemeister and G.Hommel, "Role-based Multicast in highly Mobile but Sparsely Connected Ad Hoc Networks," Proc.ACM Int'1. Symp, Mobile Ad Hoc Network and Computing, Boston, MA, Aug, 2000, pp.45-50.

[9] M. Slavik, and I.Mahgoub, "Spatial disribution and channel quality adaptive protocol for multi-hop wireless broadcast routing in vanet," IEEE Transactions on Mobile Computing, 2010.

[10] C. Plazzi, S. Ferretti, M. Roccetti, G. Pau, and M. Gerla, "How Do You Quickly Choreograph Inter-Vehicular Communications? A Fast Vehicle to-Vehicle Multi-Hop Broadcast Algorithm," in Proc. of the 4th IEEE Consumer Communications and Networking Conference (CCNC 2007), January 2007.

[11] R.Singh, and A.Gupta, "Information Dissemination in Vanets using zone based forwarding," in Proc. of Wireless Days, October 2011.

[12] T. Aguilar, S. Syue, V. Gauthier, H. Afifi, and C. Wang, "CoopGeo : A Beaconless Geographic Cross-Layer Protocol for Cooperative Wireless Ad Hoc Networks (Minor Review)," IEEE Transactions on Wireless Communications, 2011.

[13] L. Tung and M. Gerla, "Efficient Road-based Directional broadcast," in Proc. IEEE Vehicular networking Conference, Amsterdam, 2010.

[14] L. Chou and Y. Yang, "Location-Based Directional Broadcast for intervehicle Communications," in Proc.IEEE Vehicular Technology conference, Ottawa, Septembre 2010.

[15] IEEE Computer society, "IEEE standard for information technologytelecommunications and information exchange between systems - local and metropolitan area networks - specific requirements - part 11: Wireless lan medium access control (MAC) and physical layer (PHY) specifications. IEEE Std 802.11-2007 (Revision of IEEE Std 802.11-1999)," pp. 1-1184, June 2007.

[16] J. Nzouonta, N. Rajgure, W. Guiling, and C. Borcea, "VANET Routing on City Roads using Real-Time Vehicular Traffic Information," IEEE Transactions on Vehicular Technology, vol. 58, no. 7, Septembre 2009.
[17] M. Sun, W. Feng, T. Lai, K. Yamada, H. Okada, and K. Fujimura, "GPSbased message broadcast for adaptive inter-vehicle communications, "in Proc.International Conference on Parallel Processing (ICPP), pp. 26852692,2000

[18] T. Kim, W. Hong, and H. Kim, "An effective Multi-hop Broadcast in Vehicular Ad-hoc Network," in Proc. of the 5th annual ACM/IEEE international conference on Mobile computing and networking, pp. 151162 , October 2005.

[19] S. Ni, Y. Tseng, Y. Chen, and J. Sheu, "The Broadcast Storm Problem in a Mobile Ad Hoc Network," in Proc. of the Architecture of Computing Systems (ARCS 2007), March 2007. 